\title{
El dispositivo artístico. Un acercamiento crítico y genealógico a las condiciones aporéticas de la producción artística latinoamericana: el caso de la Vanguardia argentina de los años sesenta ${ }^{1}$
}

\section{The artistic device. A critical and Family to the aporetic condition of Latin American artistic production approach: the case of Argentina Vanguardia sixties}

Mtro. Emiliano GARCíA CANAL Universidad Autónoma Metropolitana (México) garciacanal@gmail.com

Recibido: 10 de septiembre 2013 Aceptado y Publicado: 24 de noviembre de 2013

\section{Resumen}

En el presente trabajo se ofrece un acercamiento reflexivo en torno a cómo el arte, en tanto producto de la cultura liberal, es ante todo un dispositivo disciplinario que ha cooptado para sí al acontecer estético y ha logrado posicionar la idea de su supuesta universalidad y autonomía.

Por tanto, se propone realizar una reflexión crítica de cómo el dispositivo artístico, fundamentado en las ideas de autonomía y universalidad, ha coadyuvado para hacer del acontecer estético una especie de propiedad privada que, por un lado, sólo el artista es

\footnotetext{
${ }^{1}$ Artículo producto de una Ponencia presentada en el VI Encuentro Panamericano de Comunicación COM PANAM 2013 celebrado la Escuela de Ciencias de la Información de la Universidad Nacional de Córdoba (Argentina), durante los días 5, 6 y 7 de junio de 2013.

El trabajo aquí presentado es producto de los avances de investigación realizados por el autor en el área de Comunicación y Política del Doctorado en Ciencias Sociales de la Universidad Autónoma Metropolitana, unidad Xochimilco, México, D.F.
} 
capaz ontológica y jurídicamente de manifestar y, por el otro, sólo el sujeto sensible, aquel que ha naturalizado la sensibilidad propia de la cultura burguesa liberal, es capaz de disfrutar para reconocer su libertad y su derecho a poseer y dominar.

Es decir, el arte no sólo cumple una función ideológica por su condición de mercancía, sino que además condiciona históricamente lo que puede ser reconocido o no como estético; y a su vez, en ese reconocimiento, propone los límites, asegurados desde la misma cultura burguesa, para determinar cuáles fenómenos estéticos son dignos de mayor valoración y procuración en la vida social. Política sobre la estética que asegura la preeminencia de lo estético a aquellos sujetos que, por "genio" o por "ingenio", tienen un supuesto derecho ontológico a la cultura.

No sólo se busca reflexionar en torno a las condiciones aporéticas propias de la producción artística en tanto mercancía de la cultura liberal, sino que dicha reflexión se ubica históricamente en uno de los ejemplos paradigmáticos de la producción artístico-política latinoamericana de la segunda mitad del siglo XX: la Vanguardia argentina de los años sesenta. La intención es dar cuenta del modo en que dicho dispositivo artístico forma y conforma consecuencias materiales contradictorias, incluso en los proyectos artísticos más contestatarios.

\section{Abstract}

In this work, a thoughtful approach about how art is offered as a product of liberal culture, is primarily a disciplinary device that has co-opted for other aesthetic events and has managed to position the idea of their supposed universality and autonomy.

Therefore, it is proposed to make a critical reflection on how the artistic device, based on the ideas of autonomy and universality, has contributed to a kind of aesthetic events of private property on the one hand, only the artist is able and legally ontological manifest and, on the other, only the sensitive subject, one that has naturalized the sensitivity of the liberal bourgeois culture, is able to enjoy their freedom and to recognize their right to own and dominate. 
That is, art is not only fulfills an ideological function for being a commodity, but also determines historically what can be recognized or not as aesthetic, and in turn, in that recognition, proposed limits, secured from the same bourgeois culture to determine which aesthetic phenomena are worthy of further evaluation and procurement in social life. Policy aesthetics ensuring the primacy of the aesthetic to those individuals who, by "genius" or " talent" have alleged ontological right to culture .

Not only seeks to reflect on the characteristics of artistic production in both the liberal culture merchandise aporetic conditions, but such reflection is located historically one of the paradigmatic examples of Latin American artistic production policy in the second half of the century XX: the Vanguardia Argentina sixties. The intention is to account for the way in which the artistic device forms and shapes contradictory material consequences, even the most rebellious artistic projects.

Palabras Clave: producción artística; latinoamericana; Vanguardia argentina; dispositivo artístico.

Key Words: artistic production; Latin American; Argentina Vanguardia sixties; artistic device

\section{Introducción: el arte como dispositivo disciplinario}

En el presente trabajo se pretende indagar sobre el acontecer del fenómeno artístico desde una perspectiva que permita ubicarlo más allá de la materialización objetivada de aquello que puede ser reconocido como "obra" de arte. Este desplazamiento del arte hacia el ámbito de lo social y político tiene el claro objetivo de reconocer lo artístico como un complejo entramado de fuerzas, no exento de tensiones, que puede ser comprendido bajo la categoría foucaulteana de dispositivo ${ }^{2}$.

2 Por dispositivo habría que entender un complejo entramado de discursos y prácticas que se entrelazan y constituyen cierta regularidad, cierta organicidad y cierta finalidad, delimitando las posibilidades del acontecer social. La familia, la escuela, la religión, la prisión, la medicina, la ciencia, 
Asumir lo artístico como dispositivo de saber/poder que ha coadyuvado en la conformación y legitimación de la "alta cultura", en tanto el único modo posible y real del acontecer cultural, implica reconocer por lo menos dos aspectos de suma importancia: por un lado, dicho dispositivo se constituye en concordancia con las relaciones de producción y de significación que son propias de las sociedades capitalistas en cada momento histórico. En ese sentido, es importante apuntar que en el complejo entramado de fuerzas que configuran al dispositivo artístico se juega siempre una dimensión política, una negociación sobre lo que puede ser enunciado -por tanto, comprendido- y materializado como artístico. Esta dimensión política en general se invisibiliza por la naturalización que ha ejercido la formación discursiva hegemónica de la autonomía y la universalidad del arte. Por otro lado, dicho dispositivo ha contribuido de diferentes maneras en los procesos de subjetivación estética, produciendo e incorporando, normando y sancionando en relación a lo que es susceptible de ser concebido y vivenciado como estético; en este punto, se hace patente que los mecanismos de naturalización que históricamente se han hecho presentes en dicho dispositivo, han postulado y jerarquizado un orden en el que se establece que la objetivación artística es la vía privilegiada para el acontecer de la experiencia estética produciéndose una segunda invisibilización: la vida, para ser vivenciada de manera estética, debe conformarse según la normalización objetivada históricamente en el arte ${ }^{3}$.

la heterosexualidad son ejemplos de dispositivos. En todos ellos se implican formas discursivas del saber que disponen y se disponen en prácticas concretas, deviniendo formas moduladas del ejercicio de poder y, por tanto, produciéndose las condiciones, por un lado, de la objetivación de las cosas que son vinculadas a la dinámica interna del dispositivo -saber/poder-; y, por el otro, de la objetivación de los sujetos -subjetivación- que socializan al interior de los distintos dispositivos. En ese sentido, lo artístico como categoría genérica de un acontecer muy diverso, en el cual se correlacionan muchos más fenómenos que el puro artefacto reconocido como "obra", es pensado aquí como dispositivo.

${ }^{3}$ Con vivencia estética se quiere hacer énfasis en las siguientes ideas: la relación sensible del sujeto con el mundo no es pasiva, por el contrario es constitutiva; las categorías históricamente relacionadas a la experiencia estética como la belleza, lo sublime, lo pintoresco, lo grotesco, entre otras, no dan cuenta de características materiales de la cosa ni psicológicas del sujeto, sino de la mutua relación; dichas categorías no agotan el universo relacional sensible que puede suscitarse en la experiencia de vida, ya que la experiencia estética se da en toda relación entre sujeto y objeto; en la vivencia 
Una primera hipótesis se hace presente: estas dos formas de invisibilización son resultado, en gran medida, del accionar del dispositivo del arte y de cómo por medio de éste se ha logrado controlar y normalizar el acontecimiento artístico y su fuerza productiva, su capacidad de resistencia, su dimensión política. Podría decirse entonces que, en tanto dispositivo, el arte cumple la misma función que todo dispositivo: disciplinar al individuo para subjetivarlo de tal manera que se potencie su capacidad productiva en términos económicos y se reduzca en términos políticos. (Foucault: 1995, p. 142) En este sentido, deja de importar la intención del autor y sus posibles pretensiones libertarias de emancipación y autonomía, pues lo que se habrá de investigar es si el arte, como discurso y como práctica, es capaz de liberarse de su propia aporía ${ }^{4}$, de su condición de dispositivo disciplinario que colabora en la regulación y normalización de la subjetivación estética y de la objetivación de la cultura.

La dimensión del problema teórico aquí planteado desborda, por obvias razones, la posibilidad del presente trabajo; por lo tanto, en las siguientes páginas se realizará un acercamiento crítico e históricamente situado del caso de la Vanguardia argentina de los años sesenta. La elección de este grupo de artistas responde a la estrategia de analizar la tensión aporética propia del dispositivo artístico, en uno de los intentos más audaces de trastrocamiento del antedicho dispositivo en la región latinoamericana. Por tanto, la intención será comprender qué tipo de subjetivación estético-política promovían, por medio de sus discursos y prácticas, estos artistas, para entonces estudiar cómo lidiaron y resolvieron las condiciones aporéticas propias del fenómeno artístico.

estética se implica siempre la mutua afección entre sujeto y objeto, de ahí la necesidad social de disciplinar la relación sensible con el mundo y el arte será el dispositivo privilegiado para normar dicho acontecer.

${ }^{4}$ La noción de aporía implica la condición contradictoria de lo artístico. Con ella se quiere plantear la necesidad de reconocer que el arte, además de ser un fenómeno que posibilita cierta dimensión para la libertad humana -idea romántica-, también cumple la función de normalización y disciplinamiento estético. Es decir, ayuda en la conformación de un tipo de subjetividad acorde a la sociedad contemporánea. 
Los años sesenta coinciden en este país, así como en varios otros de América Latina, con el desplazamiento paulatino del discurso nacionalista heredado del siglo XIX, el cual permitió la conformación y consolidación de un nacionalismo cultural identitario en la región. Asimismo, coinciden con el fervor político y artístico-cultural que siguió a la Revolución cubana y que se potencia con el mayo del 68, entre otros acontecimientos. Así también, se verá emerger un discurso cosmopolita que pugnará, sin negar lo propio, por un universalismo cultural occidental de corte capitalista que devendrá, tanto en el plano político, como en el cultural y artístico, en la conformación y legitimación de un discurso neoliberal y anticomunista.

En este escenario, el cual es todavía más amplio y complejo de lo que aquí se ha podido esbozar, el dispositivo artístico ha cumplido la función de establecer los límites de lo visible y lo pensable en términos estéticos, pero sobre todo en incorporar y subjetivar -por medio de diferentes prácticas y aún contraviniendo las intenciones de sus creadores - la idea de que lo estético es fundamentalmente no político.

Si bien, es ampliamente conocida y documentada la producción artística con intenciones y pretensiones políticas en la región -la Vanguardia argentina de los años sesenta es paradigmática en ese sentido-, es importante apuntar que el dispositivo artístico y sus discursos han podido, con el paso del tiempo, objetivar la idea de que lo político es algo que se impone a la forma desde afuera; y que, en la mayoría de los casos, dicha imposición produce panfletos que desvirtúan la condición estética y artística misma, su principio de autonomía artístico: arte por el arte. En ese sentido, en el mejor de los casos, lo político queda subsumido a la condición estética, lo que ha permitido, una vez que el objeto artístico es descontextualizado de su ámbito de emergencia e ingresado al mercado de distribución y exhibición del arte y la cultura, apreciarlo, por un lado, prioritariamente como mero acontecimiento estético y, por el otro, con suerte, como acontecimiento anecdóticamente político. El mercado ha devenido en un potente sistema de purificación que permite separar, del acontecimiento simbólico, las partículas políticas para apreciar, en dicho fenómeno, 
únicamente lo "verdaderamente" artístico. Lo político se exorciza y deviene dato histórico, curiosidad de archivista. Este problema fue, como se verá más adelante, tempranamente diagnosticado por la Vanguardia argentina, e incluso se constituyó en uno de los ejes problemáticos principales del trabajo de los artistas rosarinos y bonaerenses a finales de la década de los años sesentas.

En relación a lo anterior, pensar el acontecer artístico exige comprender cómo las nociones de arte, de artista, de autor, de público, de obra, de crítica, de forma, de técnica, de belleza, de estética, de museo, de colección, de genialidad, de originalidad, de transgresión, entre otras, conforman el entramado de lo históricamente enunciado que constituye el saber de una época sobre el arte; ámbito discursivo en el que se sustentan y fundamentan unas serie de prácticas concretas que permiten la materialización de lo artístico.

Por tanto, ubicar el presente trabajo en el contexto de la Vanguardia argentina de los años sesentas tiene el objetivo de analizar cómo se materializaron una serie de prácticas, para averiguar si dichas prácticas han coadyuvado a producir y reproducir las condiciones de la exclusión social que perseguían eliminar. Como se indicaba más arriba, el dispositivo artístico enseña cómo debe ser la vida estética y disciplina, al igual que otros dispositivos que trabajan paralelamente sobre otras dimensiones de la vida, al sujeto para que persiga y priorice cierto ámbito sensible, casi siempre mediado por alguna mercancía, como condición de "felicidad".

\section{Desarrollo: 1968, la problematización estético-política de la Vanguardia}

1968 fue un año de grandes tensiones. Múltiples ciudades del mundo fueron escenario activo de amplias movilizaciones estudiantiles y obreras, de fuertes represiones e incluso de masacres como la gestada por el Estado mexicano el 2 de octubre en la Plaza de las Tres Culturas en Tlatelolco. El ambiente político que se vivía en muchas urbes occidentales y que 
alentaba a transformar las condiciones sociales, económicas y culturales establecerá un parteaguas, un antes y un después que dejó marcadas a las generaciones venideras. Hablar del 68 es hablar de una fecha simbólica en la que se enmarca el sentir de cambio y transformación de toda una década.

En esos años, América Latina vivía, como hoy, una fuerte intervención y control por parte de Estados Unidos de Norteamérica, consecuencia de las necesidades geopolíticas hemisféricas que inauguró la Guerra fría. Las políticas estatales de los países latinoamericanos, con excepción de Cuba, privilegiaban a las oligarquías internas y a los grandes capitales extranjeros y, en no pocos casos, se impusieron, en las décadas de los sesenta y setenta, gobiernos militares para garantizar el control social y económico de la zona. Esta última circunstancia permite, sin que por eso se justifique, intuir el temor que tenían las fuerzas reaccionarias en todo el continente al grado de efervescencia social que se vivía en toda Latinoamérica tras el triunfo de la Revolución cubana.

La Vanguardia artística, al igual que muchos sectores de la sociedad argentina, también fueron copartícipes de este deseo de cambio social y cultural que se gestó durante la década de los sesenta y que alcanzará uno de sus momentos cúspides en mayo de 1969 con la revuelta social conocida como el Cordobazo, la cual gestará el final de la dictadura militar que desde 1966 encabezaba Juan Carlos Onganía.

Es en este contexto social y político, que un grupo de jóvenes artistas rosarinos y bonaerenses, hoy conocidos como la Vanguardia argentina, se preguntan por la relación entre arte y política, pregunta que los lleva a radicalizar sus formas de produción y a establecer un programa que buscaba, por medio de acciones concretas y directas, equiparar estética y política con el objeto de contribuir en la construcción de las condiciones para el cambio y la transformación social. Es decir, a diferencia del Realismo soviético o del Muralismo mexicano en los que sus artistas trabajaron como intelectuales orgánicos al servicio de las revoluciones triunfantes, la Vanguardia argentina asumió el reto de utilizar el 
arte para revolucionar la estructura social y cultural, a la que vislumbraban como anquilosada.

A finales del 68 se realiza un evento denominado Primer Encuentro de Argentina Cultura 1968: informe y debate, mismo que fue convocado por la artista Margarita Paksa y organizado en el local de la Sociedad Argentina de Artistas Plásticos. El encuentro tenía el propósito de constituir un espacio de evaluación de lo realizado y acontecido durante todo el año, así como tender los puentes necesarios con otros grupos artísticos y sociales no vinculados directamente a la Vanguardia.

Al evento acontecido los días 27 y 28 de diciembre se adhirieron varios representantes del ámbito artístico y cultural entre los que interesa destacar la participación de León Ferrari, Juan Pablo Renzi y Roberto Jacoby, quienes junto con Margarita Paksa representaban a los artistas de Vanguardia que habían protagonizado una serie de acciones orquestadas e intencionalmente dirigidas a proponer y expresar su posicionamiento sobre lo que debía ser la relación arte y política. Asimismo, como portavoces del grupo de artistas que habrían realizado la exposición Homenaje a Latinoamérica ${ }^{5}$, se presentaron los artistas Luis Felipe Noé y Ricardo Carpani.

Las palabras inaugurales corrieron a cargo de Margarita Paksa, quien ofreció una problematización puntual sobre algunos tópicos no resueltos en la conflictiva relación entre arte y política. Según Paksa:

Nuestras artes visuales, han estado dirigidas desde siempre a un público de élite, a una intermediario, el crítico, el marchand, el coleccionista, sin posibilidades de acción directa sobre el público, sino siempre [a través] de esta intermediación, la más de las veces poseedor único de nuestra obra.

\footnotetext{
${ }^{5}$ En 1967, después del asesinato del Che Guevara, se realiza una exposición en la que participó Ricardo Carpani, entre otros miembros del grupo Espartaco. La exposición fue clausurada al día siguiente.
} 
Lo que a nosotros nos toca demoler, aproximar, transformar, es el Arte, la Estética, entendidas como barreras que nosotros mismos levantamos para diferenciarnos [...] nosotros con nuestro quehacer estamos implicados, contaminados, viviendo nuestra contradicción en cuanto seres conscientes, asistiendo a una sociedad que negamos y proponiendo otra a la cual aspiramos.

Las artes en nuestra sociedad occidental, son tanto más especializadas en cuanto se encierran en sí mismas y tienen a la estética como su único objetivo. Tienden así a agudizar la alienación de la sociedad, constituyendo compartimentos estancos. (1968, p. 2)

Por su parte, en el documento leído por Ferrari también pueden encontrarse una serie de apuntes de suma importancia sobre la posición que el grupo de Vanguardia tomó ante la relación entre arte y público de élite. Interesa destacar aquí esa suerte de análisis y diagnóstico que Paksa y Ferrari ofrecen para comprender la tensión que el grupo observaba, a finales de la década de los sesentas, respecto a lo que se ha denominado en este trabajo como dispositivo artístico. Ferrari dice:

El arte, proclamado por sus autores y sus compradores como una de las más generosas y elevadas actividades humanas destinadas a enriquecer la vida de todos los hombres, es en realidad un adorno de un pequeño reducto desde el cual los grupos adinerados lanzan sus campañas de represión [...] El arte no sirve hoy para enriquecer la vida humana: sólo se usa para complacer a unos pocos y para que éstos la usen para poner la vida humana a su servicio, al servicio de las minorías cultas o seudocultas [...]

Pareciera que las clases pudientes, en su afán por diferenciarse del pueblo, en su afán por buscar un señalador que los distinga de las "masas" y que no pueda ser vulgarizado y utilizado por otros, perdiendo así su característica diferente, han fomentado a los artistas, en especial a la vanguardia, a producir los indicadores más incomprensibles, las palabras más difíciles, los signos más impenetrables para que, expuestos a la luz del día, continúen conservando ese carácter de secreta contraseña de la lógica de la cultura y del poder. (2005[1968], pp. 29 y 30) 
En estos fragmentos se realiza una descripción del vínculo entre un supuesto fin humanista emancipatorio y la relación de poder y de control que se establece y legitima, por medio del arte, entre las élites culturales y la "masa". En ese mismo sentido, Ferrari también dice:

[...] debemos tener en cuenta que "cultura" es una sucia palabra; y que la más usada de sus muchas acepciones es la que implica que cultura es una parte de los bienes que poseen algunos reducidos grupos sociales. Me refiero en especial a la cultura estética.

Me parece que hay una suerte de escondida e inconsciente complicidad entre las elites productoras de dinero y las elites productoras de cultura estética para limitar el significado de aquella palabra a lo que ellos mismos hacen, compran y usan. (2005[1968], p. 28)

Como puede verse, la crítica está apuntada a dar cuenta de la estrecha relación existente entre aquellos que producen arte y aquellos que lo consumen. Ambos se benefician del estatus que, de distintas maneras, les confiere la obra artística. Asimismo, sobre la idea de que el arte tiene la potencia de introducir un cierto nivel de denuncia o crítica social, Ferrari apunta un planteamiento poco grato pero muy potente. Para este artista existe un entramado cultural que ha logrado encasillar la producción y valoración artística únicamente sobre el ámbito formal. Esto ha permitido, según Ferrari, que se legitime la idea de que la representación estética implica una desvinculación con sus posibles contenidos, privilegiándose la forma y negándose cualquier propósito explícitamente político:

El triunfo de las obras significó el fracaso de las intenciones. La denuncia fue ignorada y el arte fue aplaudido: el arte se convirtió en el enemigo del denunciante, en el apaciguador de sus rencores, en el tranquilizador de sus ideas. Frente a esos cuadros políticos casi nadie hablaba de política, todos hablaban de arte. La denuncia es comprada por el denunciado y usada no sólo como señalador de prestigio cultural, sino también como señalador de esa tolerancia, esas libertades que, como la libertad de expresión y de 
prensa, constituyen otro de los mitos que maneja con eficacia la elite del dinero. (Ferrari, 2005[1968], p. 32)

Por su parte, en el mismo encuentro, Ricardo Carpani introduce, a nombre del colectivo reunido en torno a la exposición Homenaje a Latinoamérica, las siguientes ideas que permiten intuir una suerte de preocupación común con el grupo de Vanguardia. Según Carpani:

Nunca, en la historia de la humanidad, la situación del arte y del artista dentro de la sociedad ha adquirido un carácter tan problemático como en el presente.

Nunca, como ahora, los artistas sinceros y conscientes han experimentado como desgarradora frustración la acción de crear, hasta el punto de cuestionarse la importancia social de dicha acción y la legitimidad de su supervivencia histórica. (1968, p. 1)

Además, también para Carpani se hace presente un problema no menor sobre el estatuto de mercancía y de bien cultural que en el mundo capitalista conforma a toda obra artística.

Despojada de su función condicionadora de la emotividad colectiva, del carácter público que hasta entonces había poseído, y del contacto estable y permanente con la comunidad, la obra artística devino un objeto suntuario destinado a procurar goce estético al comprador individual, en el estrecho marco de su círculo social, o en el más estrecho aún de su vida privada. Valorizándose como objeto de cambio, como mercancía, todo lo que perdió como objeto socialmente necesario. (1968, p. 2)

Si bien, Carpani igualmente ofrece una preocupada mirada por la actualidad de lo artístico, su postura, ya antes expuesta en el Manifiesto del grupo Espartaco (2003[1959]) y en el libro La política en el arte (2011[1961]), se expresaba a favor de usar el arte como un medio de 
educación social. En ese sentido, siguiendo las directrices y planteamientos de la Escuela Mexicana de Pintura ${ }^{6}$, Carpani pugnará por un arte revolucionario que ayude a conectar la cultura con el proletariado, reactivando el vínculo comunicativo que, según el artista, el sistema económico burgués se había afanado en destruir. Para Carpani, un arte educativo y promotor de una sensibilidad ideológicamente revolucionaria era un paso necesario para la transformación. Sin embargo, el artista también advertía que un arte con estas características se enfrentaría constantemente a la fuerza que ejercen los medios de comunicación y propagación de la cultura burguesa. Y es aquí donde puede observarse una común preocupación con Ferrari por el uso de la cultura y del arte como sistemas o dispositivos de control y de legitimación de las élites económicas. Así lo expresaba Carpaní:

El control por parte de la burguesía y el imperialismo de los más poderosos medios de comunicación social actuará permanentemente como un impedimento a la difusión masiva de un arte verdaderamente expresivo de la realidad social, es decir, humana, dejando filtrar tan solo aquellas obras cuya inocuidad para el régimen constituye la mejor garantía del carácter superficial, cuando no falaz, de su contenido. (1968, p. 2)

Como se ha podido ver, entre los planteamientos expresados por Carpani y Ferrari existen preocupaciones similares; sin embargo, al mismo tiempo ofrecen una idea de cultura y de arte radicalmente distinta. Mientras que el pintor del grupo Espartaco expresa una profunda confianza sobre el papel que ha de jugar el arte y la cultura como mecanismos de transformación ${ }^{7}$, Ferrari y muchos de los artistas reunidos en torno a la Vanguardia

\footnotetext{
${ }^{6}$ Véase "No hay más ruta que la nuestra" (Siqueiros, 1945).

7 "Cuanto antes se actúe por elevar el nivel cultural de las masas, mayores serán las garantías de triunfo revolucionario y más breves los plazos que a él conducen. Demos al arte la posibilidad de intervenir activamente en este proceso, haciendo que se cumpla así un doble fin: brindar a los más extensos sectores de la comunidad el goce estético, reduciendo el abismo actual entre estos sectores y la obra artística y, al mismo tiempo, promover el cumplimiento de su papel histórico fundamental, esto es, acelerar, a través de su acción dialéctica sobre la sociedad, el desarrollo evolutivo de la sociedad." (Carpani: 2011[1961], pp. 68 y 69)
} 
consideraban que el arte y la cultura eran esencialmente burgueses y que con independencia de las intenciones de sus creadores y promotores, terminaban coadyuvando en los procesos de ideologización propios de la sociedad capitalista. En el encuentro de Cultura 68, al que ya se ha hecho mención, Ferrari cierra su intervención con las siguientes palabras:

La duda que seguramente quedará planteada en esta reunión es la siguiente: ¿sirve realmente la estética, el arte, para hacer política? ¿Sabremos nosotros usar, para otros fines, la experiencia estética que adquirimos haciendo cultura para la elite? ¿Podremos desembarazar nuestra sensibilidad de los vicios allí adquiridos para poder acercarnos a todo ese mundo de la cultura popular que la elite rechaza y hacer entonces una estética antielite que nos permita comunicarnos con los sectores sociales cuya ideología compartimos?

Me temo que la respuesta puede ser negativa, que la estética no sirva, que no sepamos usarla, que no logremos inventar otra. En tal caso, me parece, será mejor buscar otras formas de acción y de expresión. (2005[1968], p. 33)

Ferrari abre así un cuestionamiento que da cuenta de la compleja tensión aporética que constituye al dispositivo artístico: ya sea como mercancía de prestigio social, ya sea como discurso y práctica que determina la condición de disciplinamiento de la subjetivación estética, el arte ha permitido y facilitado que cierto sector social legitime su poder y gobernabilidad, entre otras cosas, por la pretendida pertenencia de un saber y sensibilidad artístico-cultural propias de las élites, es decir, de los capacitados para determinar y dirigir las riendas de la Nación.

En ese sentido, el arte en particular y la cultura en general coadyuvan en los procesos de legitimación que han permitido la dominación de las minorías sobre las mayorías. Dominación que se valida en el sentido común de que algunos son ontológicamente más humanos, pues logran sublimar, por medio de la cultura y el arte, el ámbito sensible y 
estético de la existencia, la cual, de no ser por la antedicha sublimación, mantendría a los sujetos maniatados a su condición instintiva y pasional, cercana a la animalidad. El arte y la cultura, entonces, ofrecen un prestigio social, un capital simbólico (Bourdieu) que ha permitido legitimar, a lo largo de la historia del capitalismo, la exclusión y dominación social.

Es sumamente sintomático que estas ideas ya estuvieran presentes en los cuestionamientos que la Vanguardia argentina abrió y expuso durante la década de los sesenta, pero principalmente, durante el año de 1968. En agosto de ese mismo año, meses antes de que se llevara a cabo la reunión Cultura 68 convocada por Margarita Paksa, se realizó en la cuidad de Rosario el Primer Encuentro Nacional del Arte de Vanguardia. En otro texto, ahí presentado por Ferrari, pueden anticiparse varias de las ideas que ya se han comentado, resaltando la claridad explicativa y argumental con la que se cuestiona la histórica relación de complicidad entre artistas y élites culturales.

Nuestra vanguardia, en efecto, como casi todas las vanguardias contemporáneas, dirigió sus obras a los críticos, teóricos, coleccionistas, museos, instituciones, muestras internacionales, periodísticas, etc., que los comprendían, alentaban y daban prestigio. Este primer público, al que se dirigían los artistas, esta suerte de elite cultural de la plástica, hizo entonces las veces de intermediario y explicador de las nuevas tendencias introduciéndolas en los sectores más pudientes [...]

[...] las vanguardias se dedican en general a desarrollar nuevos lenguajes cuyas claves desconocen las mayorías, desconocimiento que la induce a rechazar la obra o a mostrase indiferente, actitud que origina el alejamiento del artista quien se vuelve hacia las minorías que dicen comprenderlo. Esas minorías entonces realizan con el artista una suerte de canje de prestigios: le ofrece sus salas, sus premios, sus becas, sus publicaciones, sus fiestas, que prestigian al artista y le toman sus obras, o su amistad, que la prestigian [a la minoría] y que usa como indicadores de posición social y cultural. Es decir, que la cultura producida por los artistas que ideológicamente se sienten cerca de las mayorías populares, es usada por las minorías, en su constante búsqueda de argumentos que 
justifiquen sus privilegios, en su constante afirmar que eso que la elite entiende por cultura da derechos a poseer y gobernar. (1968, pp. 1 y 2 )

En el mismo sentido crítico que Ferrari apuntaba en el encuentro de Buenos Aires, Cultura 68, también en esta ocasión establecería un diagnóstico contundente sobre el papel que ha jugado todo artista, de vanguardia o no, con respecto a la tensa y provechosa relación de estos con las élites culturales. Su apuesta política se inscribía en procurar mensajes significativos para la mayoría, un arte de contenidos, más que de formas; si bien, el artista reconocía la complicación que esta apuesta conllevaba, en virtud de la necesidad de desembarazarse de la cultura -burguesa- misma, comprendía la necesidad de abrir la significación hacia un público históricamente excluido de la experiencia artística.

El cambio de público, el cambio de coautor, implica un cambio en la manera de analizar y estudiar una obra. El artista ya no verá más a su obra en el espejo formado por los aplausos y criticas de los teóricos de la elite, que se detienen en los aspectos formales de la misma, sino que la verá a través de las reacciones y opiniones de las mayorías, que suelen preocuparse más por lo que la obra significa que por su estilo formal.

Es decir que el artista juzgará sus obras, y las ajenas, por lo que las mismas signifiquen para el público al que van dirigidas. Las obras no son entonces geométricas, informalistas o figurativas, sino que significantes o no significantes. (Ferrari: 1968, p. 5)

Otro acontecimiento, suscitado en mayo de 1968, de suma importancia para el análisis hasta aquí presentado sobre el dispositivo artístico y sobre la condición aporética del arte, es el que protagoniza el artista Pablo Suárez en el contexto de la exposición denominada Experiencia 68, convocada por el Instituto Torcuato Di Tella que estaba a cargo de Jorge Romero Brest. Suárez, quién había sido convocado a participar en la nombrada exposición, a último momento decide declinar su invitación y realizar una acción performativa en las 
inmediaciones del Instituto. Con el fin de establecer distancia y romper relaciones con la institución cultural más importante del momento, la cual durante la primera parte de la década de los años sesenta habría albergado y apoyado la producción de muchos de los artistas reunidos en torno a la Vanguardia, Suárez decide escribir una carta dirigida al director de tan importante institución, en la que expresaba las razones por las cuales declinaba su participación en Experiencia 68. Según Ana Longoni ${ }^{8}$, el artista mandó imprimir un tiraje de 25,000 ejemplares de la carta, los cuales fueron repartidos por el propio artista durante la inauguración del evento y días subsecuentes. Asimismo, Suárez logró que los vendedores de periódicos de los kioscos cercanos al Instituto Di Tella adosaran la carta a diarios y revistas mientras duró la presentación.

Este acto fue uno de los primeros que la Vanguardia protagonizará en el proceso de radicalización de sus posturas y estrategias políticas que llevarán a la ruptura definitiva con Brest y con su instituto. En la carta comentada puede leerse lo siguiente:

Es evidente que, de planear situaciones morales en las obras, de utilizar el significado como una materialidad, se desprende la necesidad de crear un lenguaje útil, una lengua viva y no un código para las elites. Se ha inventado un arma. Un ama recién cobra sentido en la acción. En el escaparate de una tienda carece de toda peligrosidad [...]

Si yo realizará la obra en el Instituto, ésta tendría un público muy limitado de gente que presume de intelectualidad [...] Esa gente no tiene la más mínima preocupación por estas cosas, por lo cual la legibilidad del mensaje que yo pudiera plantear en mi obra carecería totalmente de sentido. Si a mí se me ocurriera escribir VIVA LA REVOLUCIÓN POPULAR en castellano, inglés o chino, sería absolutamente los mismo. Todo es arte. Esas cuatro paredes [las de la galería] encierran el secreto de transformar todo lo que está dentro de ellas en arte, y el arte no es peligroso. (la culpa es nuestra) (Suárez: 1968, p.1)

\footnotetext{
${ }^{8}$ Pueden consultarse las notas críticas que acompañan al documento digital ICAA-756351 del archivo conformado por el International Center of the Arts of the Americas at the Museum of Fine Arts, Houston. http://icaadocs.mfah.org/icaadocs/es-mx/portada.aspx
} 
La frase: "la culpa es nuestra" está presente de diferentes maneras en todos los análisis y diagnósticos que el grupo de Vanguardia realizó sobre el uso y abuso que la élite hacía del arte. En las palabras de varios de los integrantes es posible observar una preocupación real sobre la capacidad de apropiación discursiva y desarticulación política que se produce cuando los proyectos artísticos son auspiciados por el mercado cultural. En ese mismo sentido, la Vanguardia argentina tuvo la capacidad de ver que no importa que tan violenta o incómoda sea la propuesta o gesto artístico, pues en el instante mismo que algo es concebido como arte, ese algo es susceptible de ser reintegrado al sistema de intercambio de bienes materiales y simbólicos, por tanto, deviene en mercancía, y su posible significado político se desplaza a segundo plano, permitiendo únicamente la diferenciación taxonómica entre formas distintas de producción artística. Es esa la suerte implícita a toda producción que emerge del dispositivo artístico.

[...] el pequeño público y los intermediarios demostraron su exitosa capacidad de absorción tomando la obra por sus formas y minimizando sus contenidos. El arte es tan importante para ellos que si la obra es arte la denuncia desaparece. Las villas miserias con latas pegadas se vendieron a muy buenos precios y los obreros revolucionarios con puños cerrados se colgaron en los salones de los patrones. (Ferrari: 1968, p. 3)

Ante tan abrumador panorama y sin claudicar en la pretensión de realizar acciones directas que posibilitaran transformaciones sociales, la Vanguardia ideará un proyecto de denuncia social y de contra-información mediática. Habiendo comprendido que la ruta de la revolución no se daría por medio del arte, se propusieron la realización de un proyecto, que hoy llamaríamos interdisciplinario, donde conjugaron el saber sociológico y comunicativo con el estético. Dicho proyecto tenía la intención de develar la realidad de miseria y represión que se vivía en la provincia tucumana. Tucumán Arde fue la acción estético-política más radical 
que realizó el grupo, y su radicalidad se sustenta principalmente en la búsqueda de trastocar el dispositivo artístico mismo al vincularlo con la comunicación masiva.

Hartos de ese modo de ser y de pensar que Juan Pablo Renzi definía en 1966 como cultura "mermelada" y atentos ante la capacidad del dispositivo artístico de absorber y endulzar cualquier gesto artístico con pretensiones políticas, el grupo de Vanguardia definirá una nueva estrategia de lucha y producción: hacer del arte un medio de contra-información mediática que permita la denuncia y que desenmascare al discurso oficial en torno a la provincia de Tucumán.

La idea de utilizar, en el proyecto Tucumán Arde, los medios de comunicación como parte constitutiva de la estrategia artística, tiene su precedente en los planteamientos y reflexiones que años antes Eduardo Costa, Raúl Escari y Roberto Jacoby realizaron en torno al fenómeno del Happenig y la comunicación de masas ${ }^{9}$. En 1966 escribieron un documento clave en el que propusieron un arte de los medios de comunicación. Asimismo, en 1967, el mismo Jacoby escribió otro texto titulado "Contra el Happenig" y en el que expresó la siguiente idea:

[...] el viejo conflicto entre arte y política ("El arte debe reflejar la realidad", "Todo arte es político", "Ninguno lo es", etc.), que siempre se quiso superar introduciendo "contenidos" políticos en el arte, tal vez sea superado por el uso artístico de un medio tan político como la comunicación masiva. (p.130)

En este sentido, Tucumán Arde consistió en la realización de una amplia investigación sociohistórica hecha por especialistas académicos del Centro de Investigaciones en Ciencias

\footnotetext{
${ }^{9}$ Es sumamente probable que la aparición en 1964 del libro Comprender los medios de comunicación de Marshall McLuhan haya influenciado a varios integrantes del grupo de vanguardia para pensar el problema del medio como mensaje.
} 
Sociales ${ }^{10}$ y completada con entrevistas, encuestas, reportes etnográficos, registros visuales y audio visuales, carteles publicitarios, entre otros documentos producidos en su gran mayoría por el grupo mismo. Estos documentos permitían comprender con mayor profundidad la problemática social y económica tucumana, la cual se había agravado tras el cierre que, desde 1966, venían sufriendo varios ingenios azucareros. El material expuesto, tanto en Rosario como en Buenos Aires bajo el nombre de Primer Bienal de Arte de Vanguardia, permitía contrastar al discurso oficial de la época con los datos producidos en la investigación de campo. Con Tucumán Arde es posible observar con mucha claridad el cuestionamiento sobre algunos de los discursos y prácticas que conforman al dispositivo artístico: lenguaje autónomo, uso ideológico de la cultura, instituciones artísticas, originalidad, autor, colectivo, intención e interacción comunicativa y aparatos comunicativos, participación activa del espectador, relación forma-contenido, incorporación de otros saberes, entre otras. De acuerdo con Andrea Guinta:

Con Tucumán Arde la vanguardia estético-política radicalizó todas sus opciones. La experiencia fue tan intensa y, en algunos casos, traumática, que condujo a muchos de sus participantes a la conclusión de que ya no era posible pensar en la transformación de la realidad a través del arte, aun cuando éste fuera de vanguardia. Para algunos artistas, la opción fue abandonar el arte para transformar la sociedad en el terreno de la lucha política. (2008, p. 293)

La experiencia y problematización que inauguró Tucumán Arde en el ámbito del arte latinoamericano fue clausurada en su momento por la represión policiaca y militar; sin embargo, la represión más potente que este intento de acción directa y revolucionaria sufrió

\footnotetext{
10 Fueron los investigadores del Centro Miguel Murmis, Silvia Sigal y Carlos Waisman quienes facilitaron al grupo las conclusiones de su investigación sobre la crisis económica y social que azotaba a la provincia tucumana a partir del cierre de ingenios azucareros. Documento digital ICAA758479 del archivo conformado por el International Center of the Arts of the Americas at the Museum of Fine Arts, Houston. http://icaadocs.mfah.org/icaadocs/es-mx/portada.aspx
} 
fue la absorción de dicho proyecto por parte del dispositivo artístico, convertido en uno de los más importantes ejemplos de Arte conceptual latinoamericano. Como atinadamente afirmaba Pablo Suárez: "El arte no es peligroso. (la culpa es nuestra)" (1968, p. 1)

El grupo de Vanguardia argentino se disolverá en 1969. Sus miembros, de acuerdo con la directrices acordadas en el Encuentro Cultura 68, no volverán a entablar relación, por lo menos en los años siguientes, con los espacios e instituciones artísticas y élites culturales que fuertemente habían cuestionado. Por tanto, es factible plantear que el acto más radical que deviene de los planteamientos de este grupo es la negación del arte mismo, de su dispositivo.

\section{Conclusiones: No más arte, no más aporía}

La Vanguardía argentina de los años sesenta tenía muy claro que la producción artística había mantenido una muy provechosa relación con las élites culturales y, por ende, con las económicas. Dicha vínculo ofrecía a ambas partes prestigio y diferenciación. Además, para la Vanguardia, las élites habían tenido desde siempre la capacidad de transformar ese capital simbólico y cultural en una suerte de plusvalía, es decir, en condición de legitimidad de su posesión y dominación sobre otros sectores sociales.

Sobre este punto, habría que indicar que si bien, la lucha de clases se explica canónicamente sobre la base de los factores socio-económicos e históricos que rigen a las sociedades, sería prudente no dejar de advertir el importante papel que juega el arte y la cultura como dispositivos disciplinarios que coadyuvan en la legitimación de las fuerzas individualizantes sustentadas en supuestos dones, capacidades y competencias de origen natural o, incluso metafísico. Así como se presupone un dimorfismo sexual originario a la especie humana (mujer/varón), también se asume en la cultura occidental que, si bien la cultura se aprende, la capacidad de apropiarse de ella, de reproducirla y, sobre todo, de 
recrearla poéticamente en forma de arte, implica un algo especial de naturaleza biológica 0 divina que no es para todos, y que permite, legítimamente, diferenciar a los que importan de los que no, a los que deben gobernar de los que deben ser gobernados.

Es así que, lo artístico, en tanto producto de la cultura liberal, parece ser ante todo un dispositivo disciplinario de normalización que, por un lado, ha cooptado para sí al acontecer estético y, por otro, ha logrado posicionar la idea de su universalidad y autonomía. Estos dos aspectos característicos de lo artístico en la modernidad, ser el acontecer estético por excelencia y serlo de manera universal y con autonomía a otros ámbitos del acontecer humano, lejos de promover y producir las condiciones de la emancipación, han ayudado a producir y reproducir las condiciones de la exclusión social sobre la vida estética para todos aquellos sujetos que, previamente excluidos de los medios de producción de la "alta cultura", se reconocen fácticamente como incultos y, por tanto, como incapaces de un vínculo estético y creativo con la existencia.

Estudiar a lo artístico y a las condiciones aporéticas que lo conforman, implica dar cuenta, por un lado, de la fuerza productiva y transformadora que se hace presente en la póiesis creativa, pero por el otro, reconocer que cuando esa fuerza poiética es usada para producir arte, el producto de dicha fuerza queda siempre, en nuestras sociedades, subsumido al dispositivo y reducido al proceso de intercambio de bienes materiales y simbólicos.

Para la Vanguardia argentina no hubo opción, la aporía fue infranqueable incluso en su proyecto más radical: Tucumán Arde. La única salida posible fue negar al arte mismo.

\section{Bibliografía}

Bourdieu, Pierre (2010). El sentido social del gusto. Elementos para una sociología de la cultura. (A. B. Gutiérrez, Trad.) Buenos Aires: Siglo XXI Editores Argentina.

Bourdieu, Pierre (1995). Las reglas del arte. Génesis y estructura del campo literario. (T. 
Kauf, Trad.) Barcelona: Anagrama.

Carpani, Ricardo (1968). "Declaración del grupo de artistas plásticos participantes en la exposición 'Homenaje a Latinoamérica', redactada y leída como ponencia por Ricardo Carpani en 'Encuentro Cultura 68'," Diciembre 1968. Documento mecanografiado, Buenos Aires: Fundación Ricardo Carpani. Recuperado de http://icaadocs.mfah.org/icaadocs/ELARCHIVO/RegistroCompleto/tabid/99/doc/762492/langu age/es-MX/Default.aspx

Costa, Eduardo, Raúl Escari y Roberto Jacoby (1966). "Un arte de los medios de comunicación", Buenos Aires: Archivo personal de Roberto Jacoby. Recuperado de http://icaadocs.mfah.org/icaadocs/ELARCHIVO/RegistroCompleto/tabid/99/doc/750362/langu age/es-MX/Default.aspx

Carpani, Ricardo (2011[1961]). La política en el arte, Buenos Aires: Continente.

Ferrari, León (1968). "El arte de los significados," agosto 1968. Documento mecanografiado. Rosario: Archivo personal de Graciela Carnevale. Recuperado de http://icaadocs.mfah.org/icaadocs/ELARCHIVO/RegistroCompleto/tabid/99/doc/755986/langu age/es-MX/Default.aspx

Ferrari, León (2005[1968]). "Cultura” en León Ferrari, prosa política, Buenos Aires: Siglo XXI Editores Argentina.

Foucault, Michel (1999). Michel Focucault, Estética, ética y hermenéutica. (A. Gabilondo, Trad.) Barcelona: Paidós.

Foucault, Michel (1995). Vigilar y castigar-. nacimiento de la prisión, (A. Garzón del Camino Trad.), México: Siglo XXI.

Giunta, Andrea (2008). Vanguardia, internacionalismo y política: arte argentino en los años sesenta, Buenos Aires: Siglo XIX Editores Argentina. 
Grupo Espartaco (2003[1959]) "Por un arte revolucionario" en Rafael Cippolini, ed. Manifiestos argentinos. Políticas de lo visual, 1900-2000, Córdoba, Argentina: Adriana Hidalgo Editora.

Jacoby, Roberto (1967). “Contra el happening”, en Oscar Masotta (comp.). Hapepenings, Buenos Aires: Jorge Álvarez.

Longoni, Ana y Mariano Mestman (2008). Del Di Tella a "Tucumán Arde. Vanguardía artística y política den el 68 argentino”, Buenos Aires: Eudeba.

Paksa, Margarita (1968). "Texto del informe de Margarita Paksa a Cultura 1968", Documento mecanografiado, Rosario: Archivo personal de Graciela Carnevale. Recuperado de http://icaadocs.mfah.org/icaadocs/ELARCHIVO/RegistroCompleto/tabid/99/doc/761355/langu age/es-MX/Default.aspx

Renzi, Juan Pablo (1966). A Propósito De La Cultura Mermelada. Manifiesto, Rosario: Archivo personal de Graciela Carnevale. Recuperado de http://icaadocs.mfah.org/icaadocs/ELARCHIVO/RegistroCompleto/tabid/99/doc/753432/langu age/es-MX/Default.aspx

Siqueiros, David Alfaro (1945). No hay más ruta que la nuestra: Importancia nacional e internacional de la pintura mexicana moderna. México: Talleres Gráficos de la Secretaría de Educación Pública. Recuperado de http://icaadocs.mfah.org/icaadocs/ELARCHIVO/RegistroCompleto/tabid/99/doc/774358/langu age/es-MX/Default.aspx

Suárez, Pablo (1968). Carta mecanografiada a Jorge Romero Brest, Buenos Aires, mayo 13, 1968. Buenos Aires: Archivo Di Tella, Universidad Di Tella. Recuperado de http://icaadocs.mfah.org/icaadocs/ELARCHIVO/RegistroCompleto/tabid/99/doc/756351/langu age/es-MX/Default.aspx 


\section{Forma de citar este artículo en bibliografías}

GARCÍA CANAL, E. (2013): "El dispositivo artístico. Un acercamiento crítico y genealógico a las condiciones aporéticas de la producción artística latinoamericana: el caso de la Vanguardia argentina de los años sesenta”, en Revista PANGEA, Vol. 4 № 2, páginas 01 a 25. Red Académica Iberoamericana de Comunicación. Recuperado el de de 2 de: http://revistapangea.org 